\title{
A REVIEW OF EYE DISEASE IN CENTRAL CHINA*
}

BY

\author{
G. H. Pearson \\ FROM THE METHODIST HOSPITAL, \\ SHAOYANG, HUNAN, FREE CHINA
}

A RESIDENCE of 24 years in this little-known part of Free China, during which time ophthalmic work has been one of the writer's main interests, is the basis for this review.

The hospital which has grown up at Shaoyang, Hunan, during these years now has 120 beds, and a daily out-patient clinic of up to 200 patients. Of these usually 25 per cent. have been eye cases. They come to us mostly direct from peasant homes, and never having seen any other doctor. Many travel great distances along the old paths amongst the rice-fields. The climate in this part of China is moist and sub-tropical, with just an occasional winter frost.

Owing to the conditions of practice, any opportunities for laboratory work have been few, apart from a routine smear. The

- continuity of our records has frequently been interfered with by - wars and rumours of wars, which caused sudden evacuation on at least three separate occasions. Nevertheless, records which survive show that between 1929 and 1942 we treated 1,341 in-patients suffering from trachoma. We did 1,027 operations for entropion, and 51 operations for catarạct, etc. These are a sample of the numbers of casès treated.

The great bulk of our cases have always been due to the conjunctival infections and their sequelae. The treatment of diseases of the fundus, and even the prescription of glasses, have assumed a minor rôle in our work, due to local social conditions. The call for properly adjusted glasses is, however, increasing and it is now possible to get lenses ground locally by Chinese firms who understand the usual prescriptions. Amongst students especially there is a high proportion of severe myopia, often hereditary. Presbyopia has for centuries past been known and treated in China by the use of convex spherical lenses. - One just goes to a shop and asks for the appropriate glasses for a person $35,-40 ; 50$ or 60 years old, and a crudely ground lens of approximately right strength is forthcoming! Lenses of natural rock-crystal still fetch much more money than those of "mere glass"! Glasses prescribed in the old way for myopia are less satisfactory, due to the crude make of the lenses and the absence of any means of correct adjustment. Astigmatism, which is frequent, is left entirely untreated by the old methods. Strabismus of various types oceurs, but there seems to be no demand for treatment of this symptom.

* Received for Publication, October. 24, 1944. 


\section{A.-Conjunctival infections .}

1. Acute conjunctivitis of a type locally known as "Ho Yen" or "Fire Eye" is very common. The main symptom is a redness of sclera-conjunctival area, with soreness and stickiness of the lids. The bright red colour is very noticeable. 'It usually clears up under simple treatment in two or three days, or. if untreated it may lead on to corneal ulceration, etc. It is also possible that some of these cáses exhibit a transient temperature. A smear usually shows a few bacilli. The condition is very infectious, large scale epidemics are seen frequently in local schools, and amongst soldiers.

Our usual treatment is by four-hourly saline or boric washes, followed by mild antiseptic drops, as mercurochrome 1 per cent. or acriflavine $1 / 1,000$. A bandage is forbidden, If ulcer supervenes, then atropine is added to the treatment.

2. Trachoma. "Trachoma is one of China's most pressing public health problems. It is so common everywhere that it cannot possibly be tackled by eye specialists alone. "What can the 20 or 30 specialists do amidst a population of $450,000,000$ people of whom every fourth person is infected? Every doctor and every trained nurse should be instructed in its diagnosis, cure and prevention.

Diagnosis.-Possibly trachoma, as seen in China, is not quite the same as trachoma as 'seen in Egypt or in Europe. To me in Hunan any case of chronic conjunctivitis is a suspect case of trachoma and must be treated as such: I can never tell which case of sub-acute conjunctivitis is going to become trachoma. Only after some weeks can I tell. By that time the case has either been cured, when I say'it was not trachoma, or has gone on to become a typical text-book case of trachoma. So'far as one has been able to get the history of these cases, they all begin as a very mild conjunativitis, which only later becomes recognisable for what it.is. There is never any pus formation. My first question therefore to every patient is " "How long have you had it?" A non-pưrulent sticky conjunctivitis which has lasted over three weeks is considered to be a case of trachoma, and is labelled as such.'

Among the cases so labelled we may then proceed to separate out :-

1. The early case, with only rudimentary follicle formation and with as yet no macroscopic pannus formation.

2. The fully developed trachoma having many soft lymphoid follicles in the upper fornix, and over the surface of the tarsal plate. They are also often seen in the lower fornix. In these cases pannus is usually becoming well developed in the upper half of the cornea. 
3. The cicatricial-case where the follicles have mostly disappeared and where scar tissue has taken their place. These cases usually show smooth white scar tissue in the fornix covered by epithelium, but this process taking place in the tarsal plate gradually produces incurving of the lids and the formation of entropion, with further sequelae of ulceration. At this stage we often see very intense pannus which covers and obliterates the whole cornea, and is quite impervious to treatment.

4. Casies occur which I like to call, "the hard type of trachoma." In these cases the fibrous tissue; instead of being smooth and soft as above, takes on a hard nodular condition, so that the whole inner surface-of the tarsal plate area becomes covered by hard fibrous nodules, separated from each other by deep trenches of conjunctival epithelium, but so closely pressed together and flattened on the surface by friction over the cornea, that they look like a tesselated pavement when fully developed. These are probably the casés called "spring catarrh" in our textbooks. To me in Hunan they definitely belong to the trachoma family.

5. A fifth development in our cases may be the formation of a plasmoma. This is a tumour of the conjunctiva apparently reported only from China. Clinically to the writer this appears to. be a late degenerative condition, in an eye which has for long years been irritated by trachoma or cónjunctival infections. It consists of a soft easily bleeding and friable tumour mass, lying directly under the conjunctival epithelium and often involving the whole conjunctiva. It is not encapsulated and can be scraped out with a sharp spoon, rather like herring roe. So treated it shows no malignant returns, but the scarring and loss of conjunctiva may be serious.

6. In China a pterygium very often is a late complication of the multiple irritations of trachoma, though it may in theory occur without trachoma.

In such a practice as mine in China it is necessary to regard any case which shows definite follicle formation and in which the follicles can be expressed between the finger nails as " trachoma." The development of pannus (that is of macroscopic blood veśsels, first in the upper half of the cornea, then all over, finally becoming a definite layer of vascular scar tissue over the whole corneal surface) seems to bé a secondary development, due possibly to the rubbing and irritation of the corneal surface by the inflamed conjunctiva, though it is regarded by some as a direct lesion produced in the cornea by the infecting tissues.

Treatment of trachoma cases.-The lymphoid follicles when once formed may continue to grow with the continued progress of the disease, or may regress as the case goes on to a spontaneous 


\section{Eye Disease ln Central China}

cure. Certain it is that if these soft follicles are expressed, and a regime of mild washes with slightly caustic drops instituted, improvement is at once definite, although of course scar tissue and new iblood vessels do not disappear. Expression of the follicles is usually done in the O.P.D. between one's finger nails, or if preferred between the ends-of two ordinary scalpel handles. In China Knapp's roller forceps is quite useless. It is far too big and bulky to get it into these small much scarred conjunctival fornices. Anaesthesia is hardly ever used. We have no time for a general anaesthetic, and cocaine drops are of little use in such inflamed conjunctivae. At the time of expression, -and also after a subsequent wash, a drop of protargol 1 per cent. is instilled. The patient is told to attend daily for about two weeks. - The conjunctiva is rubbed over gently at each daily visit after dropping in the protargol, or if it is a badly inflamed case substituting silver nitrate 1 per cent. for the first few days (or copper sulphate 1 per cent.). The patient is told to wash his eye three or four times daily with boric lotion or normal saline, and to instill a drop of protargol 1 per cent. at night. There is no special virtue about these oldfashioned drops, but they do their job. I believe that there must be many other drugs which would have an equal effect, but they must be very slightly irritant as well as mildly antiseptic.

Note.-Even 1 per cent. protargol by itself is a slight irritant. If dropped at night into a healthy eye, it always produces a small discharge of mucus by the next morning. A patient might think that this was a sign that his eye is unwell, but in reality, such a slight discharge is simply dưe to the reaction by the healthy conjunctiva to the presence of the protargol, and it is an indication to the physician as to how these drugs produce their good effect. In my student days in England I had been taught never to use such " painful caustics as silver nitrate, copper sulphate," etc., in " the human eye. My teacher was a highly respected member of his profession, but, alas, I now use them regularly. This for two good reasons : 1 . The chronically inflamed eyes with which I am dealing are not sensitive to pain in the way that a normal healthy eye is; 2 . I find that these drugs definitely do clear up these cases, when used as I have described. Only very rarely do I find it needful to apply a copper stick or crystal with the idea of hastening. the process of cicatrisation.

Note.-The use of sulphanilamide. To me it is irrational to give sulpha drugs by mouth in these cases. We are presumably dealing with a virus disease, and viruses are not usually subject to sulphanilamide or its congeners. If, however, we have before us a late case of trachoma which has gone on to corneal ulceration and secondary infection, thẹn sulphanilamide does have a place; but it should be applied locally. : Why poison the whole body 
when it is possible for us to get the action and absorption by-local application? In this matter I have been working under some handicap, as during the past two years, we in Free China have been entirely cut off from all access to scientific printed matter from Europe or America, due to the blockade of China by Japan. I did, however, see one or two of the earlier articles, and was encouraged to experiment with the sulphanilamide, which was the only suitable preparation obtainable locally. I therefore began to dust pure sulphanilamide powder, every four hours, into the eyes of my patients suffering from any of the conditions for which it was said to be effective. So used it is slightly irritating at the time of application but the pain soon passes off. In trachoma cases which had gone on to ulceration of the cornea the results were very well worth while, and also in old pneumococcus ulcers, in acute gonococcus infections, etc. So soon as improvement had set in I changed the treatment to saline washes with atropine as required. Actually I should now prefer one of the newer soluble sulpha preparations which might be expected to allow of adequate absorption without the accompanying irritation.

Other treatment.-In the " hard type cases "I find all the above treatments useless. In these cases the patient suffers much from the chronic local irritation of the hard nodules rubbing on the cornea Even the application of crude caustic has little effect on these cases and I believe that the only satisfactory treatment is the actual excision under anaesthesia of the whole diseased area, suturing the healthy conjunctiva from the upper fornix down to the edge of the wound. These are the only cases which need such radical cure; and they are not a large proportion of the whole.

Entropion.-Under this term I include all cases, sómetimes known as trichiasis, etc., where one or many of the eyelashes has become turned in so as to scratch the cornea at each movement of the lid. In our clinic 99.9 per cent. of these cases are due to cicatrisation and contraction of scars left in the tarsal plate by the growth and healing of trachomatous lymphoid follicles. In our general medical and surgical clinic, these cases are more numerous than any other clinical entity! The scar tissue is actually in the tarsal plate itself, which therefore becomes curved when the scar tissue contracts. Often a slight degree of this condition may be more hurtful than when all the eyelashes are resting in a quiet row against the cornea; because the single hair frequently digs its point into the cornea, thus rapidly producing an ulcer. In treating' such large numbers of entropion patients one's treatment must be as rapid and as certain as possible. After actually trying out most of the available operations, we now use almost uniformly the Hotz operation with individual slight variations to fit it to each case. Half per cent. Novocain is in jected into 
the lid, a strip of skin of the desired size is removed together with the 'underlying connective tissue in one piece, thus leaving the yellow tarsal plate showing below. A narrow wedge is then rapidly excised along the whole breadth of this plate, the underlying cornea being meanwhile protected by a plain metal shield. Stitches three or four in number are inserted, including in them if necessary the fibrous tissue on the surface of the tarsal plate. At the end of this operation the lashes should be already in their final position. A dressing is applied and the eye bandaged. On the third day, i.e., after two nights, the bandage is removed, the wound smeared with vaseline and left unbandaged. On the fourth day the stitches may be removed and on the fifth the patient leaves for home cured. Time is money for these cases. They cannot afford to stay for longer grafting operations and the like. We have been getting -uniformly good results from this treatment, recurrences are almost unknown, or if they do occur, it is because the original trachomatous process is still active and fresh scars have produced a fresh deformity of the tarsal plate. A further operation plus the treatment of the trachoma will cure the case. In pioneer up-country work this is an immensely satisfying little operation, especially amongst patients who are so nervous about any risk to life. One man with very little equipment can do the operation and the patient is rescued from probable blindness for life. He is duly grateful.

Pterygium.-Although not usually listed as a trachoma complication, the many cases of pterygium that we see occur almost universally in old chronic trachoma patients. Fully developed it consists of a triangular mass of vascular fibrous tissue, its apex adherent more or less to the centre of the cornea, and having a line of adhesion along one radius of the cornea until it spreads out on to the bulbar conjunctive as its base. The conjunctiva covers the whole thing except along the actual line of adhesion to the'cornea. As seen here its development often begins as a small marginal ulcer. Small blood vessels grow towards the ulcer from the sclera, thus healing its outer edge. But as the cornea is avascular, the ulcer progresses centripetally towards the centre of the cornea, and the healing edge of blood vessels thus gradually follows the ulcer to the centre of the cornea, dragging with it as it goess its own covering of conjunctival epithelium. Treatment consists of treating the trachoma and ulcer if still unhealed, or any other irritating condition, such as smoky fires. We may remove the scar tissue at leisure, taking care to heal the new ulcer left by the incision so that it does not again repeat the circumference-to-centre scarring process. Such a patient must be warned before operation that the vision after operation will not be as good as before the disease process set in, due to the destruction of the surface epithelium of the cornea: 
Trachoma (with its sequelae) is thus one of the greatest causes of ultimate blindness in Free China. Pannus, leucomata or scars following ulcers or, entropion, and even perforation and loss of the eye are what we see in these people who are constantly coming to our clinic to see if there is anything to be done for them. I have even seen a case of mental disease as a sequela of trachomà. That was a boy with acute trachomatous disease which healed quickly but there had been several small abrasions in the corneal surface, which healed without any formation of fibrous tissue and without replacement of the small amounts of corneal tissue lost. The result was greatly reduced visual acuity due to the irregular refracting surfaces. The patient was a young student, and he was never able to accommodate himself to a life in which study and the reading of books was impossible.

\section{B.-Other diseases}

Apart from trachoma and conjunctivitis the following are alyays to be seen at our Shaoyang clinic.

1. Syphilis.-This is very prevalent amongst the whole population and is almost untreated: Whole villages of men, women and children seem to be affected, but it is certain that in many cases the course of the disease is not severe, A primary sore may be seen on the face or eyelids. Secondary or early tertiary disease shows itself as an acute iritis, which often goes on to blindness due to iritic adhesions and consequent secondary glaucoma. In children and young people keratitis punctata is frequently seen, beginning as a small pyramidal area of greyness in the lower half of the cornea. This consists of fine punctate spots if seen in a good light. There is also some redness and photophobia. It readily yields to anti-syphylitic treatment plus atropine drops. Amongst adults with late secondary or tertiary disease we see many cases of acute neuro-retinitis, -which cause a gradual loss of sight without any external signs of disease. Diagnosis may be confirmed by the ophthalmoscope. The disc at first appears red and inflamed, later it is a clear cut white patch. At that stage the eye is already blind. If seen early vigorous anti-syphylitic treatment may be effectual in saving what sight still remains. Alas, in China there is no governmental provision of arsenicals and the ordinary price of these drugs on the market is prohibitive to very many of our patients. We have to do what we can for them. Such cases are sometimes very tragic, for the patient and his friends can see nothing wrong to account for the loss of sightmany Chinese soldiers have succumbed to this condition.

2. Gonorrhoea.-In this part of China the gonococcus is always with us. In the eye department we see it first as a frequent acute purulent conjunctíitis affecting adults by direct infection 
from the genitalia. The eyelids become swollen and very painful so that the patient cannot open his eyes, and the conjunctiva is full of pus. If not attended to, the disease very early ulcerates the cornea and blindness may be complete in a day or two. This is often a soldier's disease, we have also had cases amongst careless part-trained male nurses.

The other gonococcal eye condition that troubles is ophthalmia neonatorum. The condition is preventable if the usual precautions are taken at the time of birth. (Immediate cleansing of the the eyes by the midwife, plus instillation of 1 per cent. silver nitrate drops.) Alas in China most children are born in their village homes with only the dirtiest of old women in attendance. Local superstition forbids the mother to move from her home for any cause before the baby is one month old. Such an infected child is thus usually quite blind from corneal ulceration long before it gets to hospital. Almost daily I have been seeing such babies brought up to my eye clinic! Such a blind child is not wanted. I believe that such early blindness is, usually the direct cause of the death of these children in the early months of their life, due to deliberate or involuntary neglect of the child by its disappointed parents. Our reaction to this situation as an eye clinic has been to sponsor the provision of increased midwifery teaching and accommodation.

Treatment.-In all these gonococcal cases we are now using sulphanilamide powder, as described under trachoma above. In addition we use the frequent boric or saline wash, and the continuous application of cold to the lids, because in vitro the gonococcus is very suscêptible to the effects of a cold temperature and cannot grow below blood heat. No bandage is allowed, and the pus is washed away at least every hour, the sulphanilamide being applied four hourly. In one case we attempted to use a sulphanilamide ointment, but it failed badly due to the inability of the nurses to get the ointment under the swollen edges of the lids.

3. Measles is kery common in Hunan, and it produces not a few eye cases for us. The initial lesion is an acute conjunctivitis with photophobia. Such eyes, if neglected and not washed occasionally, easily develop a small corneal ulcer, which leads on to a leucoma scar or " white cloud"' in the cornea. Very many children with severe measles recover from the disease itself after, two or three weeks but are left by it in a condition of extréme weakness and disability.' It is in these cases of extreme debility and under-nourishment after measles, that we frequently get cancrum oris, or noma. Others of them become true cases of keratomalacia and get acute massive ulceration of the cornea. If we get these cases soon enough we try to treat the underlying 
condition, giving cod liver oil and vitamins by mouth: Atropine and cod liver oil dropped directly into the eyes are also useful. Alas, in many of these children, by the time that the general condition has improved, the eyes have already become blind.

4. Smallpox-In these cases we frequently see $\mathrm{a}^{*}$ smallpox pustule on the cornea itself. Such a pustule leaves a very dense white leucoma. Treatment is on general lines only.'

5. Acute cerebro-spinal meningitis is frequent, and has been seen to cause blindness due to a spread of the infective process along the optic nerve.

6. Tuberculosis in all its forms is very frequent in this region, but we see so many other gross superficial eye lesions, that it is not often that one feels justified in making a certain diagnosis of tubercular eye trouble.

7. Senile cataract, and primary glaucoma both occur, but neither is common.

8. Accidents of all kinds trouble us. Most troublesome are the neglected cases of perforating wounds of the eye with prolapse of iris, which come for treatment days or months later. We thus frequently get cases of sympathetic ophthalmia. Our advice to these cases is complicated by local superstition; and by the fact that formér anti-foreign propaganda used the slogan that " foreign doctors gouge out your eyes to make their medicine of them." Because of this we have still to move carefully. In a case where enucleation of the wounded ot exciting eye may be expected to save the sight of the remaining eye, we explain all the circumstances to the patient, and to his relations, and get them all to sign a document saying that they understand the case, and that we may do what is necessary. Our young trained Chinese nurses are a great help in getting these explanations put through. More and more in recent days we are finding such difficulties are growing less, and our patients are "becoming willing, to accept the advice given to them.

\section{REFERENCES}

SHERE and SONDERS, B. F.-Arch. Ophthal., Vol. XXV, pp. 1025-1031. Lancet, 1939-40, quotation mislaid.

BoldT.-Trachoma, H. and S., 1904. 